\title{
Ageing memory and glassiness of a driven vortex system
}

\author{
XU DU' ${ }^{1}$, GUOHONG LL11, EVA Y. ANDREI ${ }^{*}$, M. GREENBLATT² AND P. SHUK² \\ ${ }^{1}$ Department of Physics and Astronomy, Rutgers University, Piscataway, New Jersey 08855, USA \\ ${ }^{2}$ Department of Chemistry, Rutgers University, Piscataway, New Jersey 08855, USA \\ *e-mail: eandrei@physics.rutgers.edu
}

Published online: 21 January 2007; doi:10.1038/nphys512

Many systems in nature-glasses ${ }^{1-11}$, interfaces ${ }^{12}$ and fractures ${ }^{13}$ being some examples-cannot equilibrate with their environment, which gives rise to novel and surprising behaviour such as memory effects, ageing and nonlinear dynamics. Unlike their equilibrated counterparts, the dynamics of out-ofequilibrium systems is generally too complex to be captured by simple macroscopic laws ${ }^{1}$. Here we investigate a system that straddles the boundary between glass and crystal: a Bragg glass $^{14,15}$, formed by vortices in a superconductor. We find that the response to an applied force evolves according to a stretched exponential, with the exponent reflecting the deviation from equilibrium. After the force is removed, the system ages with time and its subsequent response time scales linearly with its 'age' (simple ageing), meaning that older systems are slower than younger ones. We show that simple ageing can occur naturally in the presence of sufficient quenched disorder. Moreover, the hierarchical distribution of timescales, arising when chunks of loose vortices cannot move before trapped ones become dislodged, leads to a stretched-exponential response.

Glassy states of matter abound with seeming contradictions: macroscopically they are rigid like crystals, but microscopically their structure is closer to that of liquids. At the same time, their response to external drives is unlike that of either crystals or liquids, showing metastability, hysteresis and nonlinear dynamics ${ }^{1}$. In recent years the glass family has expanded to include systems that can be modelled by elastic manifolds in random potentials, such as vortices in superconductors ${ }^{14-21}$, domain walls ${ }^{12}$ or twodimensional electron layers ${ }^{5,6}$. When the random potential is weak these systems are expected to form a marginal glassy state, a 'Bragg glass', which is topologically ordered like a perfect crystal, but unlike crystals has no long-range spatial order ${ }^{14,15}$. An intriguing and enduring puzzle associated with this phase is the dynamics at the onset of motion: does it move as a rigid object or break up into pieces; does it crystallize at high velocities or retain its glassy nature ${ }^{22-25}$ ?

To probe the dynamics, we focused on vortex states in single crystals of $2 \mathrm{H}-\mathrm{NbSe}_{2}$ because in this material quenched disorder can be sufficiently weak to allow the formation of a Bragg glass. The vortex states were prepared by field cooling the sample below the superconducting transition in a field of $0.2 \mathrm{~T}$ and temperatures down to $4.2 \mathrm{~K}$ (see the Supplementary Information). The results reported here were obtained on a sample of size $4.4 \times 0.8 \times 0.006 \mathrm{~mm}^{3}$ and transition temperature $7.2 \mathrm{~K}$ (see the Supplementary Information). At low temperatures $(T<5.7 \mathrm{~K})$, where the Bragg glass is expected, the response of a freshly prepared field-cooled lattice to a current pulse was previously ${ }^{19}$ found to fit stretched-exponential, or Kohlrausch-Williams-Watts (KWW) time dependence ${ }^{10,11}$, spanning three decades in time: $V(t)=V_{1}\left\{1-\exp \left(-\left[\left(t-t_{0}\right) / \tau\right]^{\beta}\right)\right\}$. Here $V_{1}$ is the saturation voltage, $t_{0}$ the delay time before a measurable voltage appears, $\tau$ the rise time and $\beta \sim 0.6$. The experimental protocol consists of applying a first current pulse of amplitude $I_{1}$ followed by a second pulse $I_{2}$, during which the evolution of the voltage is recorded (Fig. 1b, inset). The pulses are separated by a waiting time $t_{\mathrm{w}}$ without current. Remarkably, the response is significantly slower during the second pulse than during the first pulse and its evolution depends not only on the elapsed time from the onset of $I_{2}$, as is the case in ergodic systems, but also on $t_{\mathrm{w}}$, so $V(t)=V\left(t, t_{\mathrm{w}}\right)$. This behaviour, also known as ageing, is one of the hallmarks of glassy dynamics $^{1-8}$. The response curves for $I_{2}=I_{1}$ and several values of $t_{\mathrm{w}}$ are presented in Fig. 1a. When the same data are re-plotted against the scaled time $t / t_{\mathrm{w}}$ (Fig. 1b), all the curves collapse without adjustable parameters onto a master curve,

$$
V(t)=V_{1}\left\{1-\exp \left(-\left[\frac{t}{\gamma t_{\mathrm{w}}}\right]^{\beta}\right)\right\} .
$$

The scaling constant, $\gamma$, is independent of $t_{\mathrm{w}}$, leading to a special and rare form of ageing, $V\left(t, t_{\mathrm{w}}\right)=V\left(t / t_{\mathrm{w}}\right)$, also known as simple or full ageing ${ }^{6-8}$. Simple ageing is remarkably robust in this system, extending over almost five decades in reduced time and holding to the longest measurement times $\sim 2 t_{\mathrm{w}}$. For $T<5.5 \mathrm{~K}$ and at low saturation voltages, $V<5 \mu \mathrm{V}$, the exponent $\beta$ is independent of $t_{\mathrm{w}}$ and temperature. Its value, $\beta \sim 0.24$, obtained for $V_{1}=1.0 \mu \mathrm{V}$, decreases slightly with increasing $V_{1}$ (Fig. 1c). Simple ageing continues to hold at higher drives, but the range of the KWW fit is reduced. We find that the KWW function fits the data over a wider range than other simple choices. For example, a logarithmic fit, also commonly used ${ }^{6}$, is indistinguishable from KWW for $t<0.1 t_{\mathrm{w}}$, but becomes worse at longer times. We note that for the second pulse $\beta \leq 0.24$ is significantly lower than in the first-pulse case, where $\beta \sim 0.6$. As shown below, this provides an important clue to the glassy dynamics of moving vortex states.

To study the case $I_{1} \neq I_{2}, I_{1}$ was varied while keeping $I_{2}$ constant. The response is a sensitive function of $I_{1}$ : it is slowest for $I_{1}=I_{2}$ and becomes faster whenever the two levels are not equal (Fig. 2a). In other words, the system retains an imprint of $I_{1}$, which can 

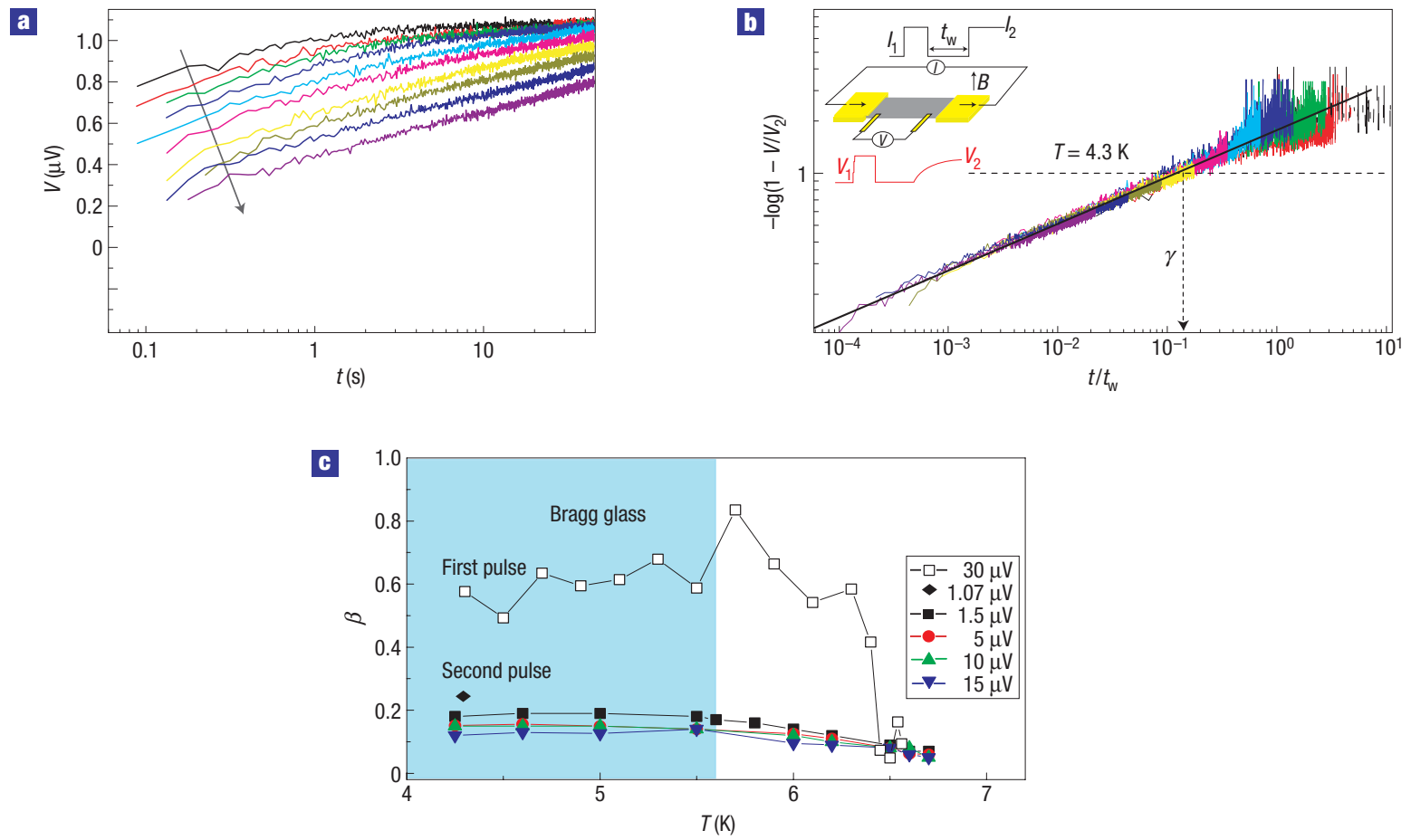

Figure 1 Ageing of the vortex lattice. a, Response during the second pulse following a first pulse of duration $t_{1}=512 \mathrm{~s}$ and amplitude $l_{1}=I_{2}=5.36 \mathrm{~mA}$. The waiting times $t_{\mathrm{w}}=4 \mathrm{~s}, 8 \mathrm{~s}, 16 \mathrm{~s}, 32 \mathrm{~s}, 64 \mathrm{~s}, 128 \mathrm{~s}, 256 \mathrm{~s}, 512 \mathrm{~s}, 1,024 \mathrm{~s}$ and 2,048 s increase along the arrow. $\mathbf{b}$, Scaled second-pulse response versus scaled time. A linear fit gives $\beta$ (slope) and $\gamma$ from the intercept, $-\log \left(1-V / V_{1}\right)=1$. The experimental set-up is shown in the inset. c, Temperature dependence of $\beta$ for first pulse and second pulse (open and solid symbols). Pulse amplitudes were adjusted to give the same saturation voltage at all temperatures.

be retrieved later in the form of a maximal slow-down during $I_{2}$. For $t>0.1 \mathrm{~s}$, the response during $I_{2}$ fits a generalized form of equation (1):

$$
V(t)=V_{2}\left\{1-f \exp \left(-\left[\frac{t}{\gamma t_{\mathrm{w}}}\right]^{\beta}\right)\right\} .
$$

Here $V_{2}$ is the second-pulse saturation voltage and $f=f\left(V_{1} / V_{2}\right)$ is a 'memory function'. As shown in Fig. 2b, for each $V_{2}$ there exists a unique value $f$ that collapses the response for all $V_{1}$ onto one curve. We plot the memory function obtained by this procedure in Fig. 2c. Although the asymptotic response ( $t>0.1 \mathrm{~s})$ obeys equation (2), this form is not valid at short times (Fig. 2d).

We studied another limit, $t_{\mathrm{w}}=0$, by applying 'step pulses' where the first pulse $I_{1}$ was directly switched after a time $t_{1}$ to the second pulse $I_{2}$. If we do not allow the response to saturate during the first pulse, the second-pulse response is identical to that of a single pulse of amplitude $I_{2}$ with a shifted time origin: $V\left[t-\left(t_{1}-\delta t\right)\right]$. The shift $\delta t$ is linear in $t_{1}$ (Fig. 3a, inset), a behaviour that provides an additional clue to the mechanism underlying the glassy dynamics in this system.

KWW relaxation is far more common than the 'conventional' exponential form $(\beta=1)$. It occurs in complex systems where the dynamics is governed by a statistical distribution of relaxation times together with constraints that restrict the path towards steady state to a hierarchical sequence of steps ${ }^{9-11}$. The hierarchy arises if certain segments (here chunks of vortices) cannot start moving until the ones in front of them are dislodged. A model of hierarchically constrained dynamics that leads to a KWW response with $\beta=1 /\left(1+\mu_{0} \log 2\right)$, where $\mu_{0}$ is the number of degrees of freedom involved in initiating the process of relaxation, has been proposed ${ }^{9}$. Thus different values of $\beta$ imply qualitative differences in the initial conditions, with smaller $\beta$ corresponding to more entangled states, which require more steps to reach steady state. The exponents, $\beta \sim 0.6$ and $\beta \sim 0.2$, imply that the corresponding initial states for the first pulse and second pulse are inherently different. For the former, $\mu_{0} \sim 1$ implies that the initial field-cooled state is readily set in motion, whereas for the latter, $\mu_{0} \sim 10$ indicates that the moving state (the second pulse is applied after the system experienced motion) is more entangled. This striking difference, together with the fact that the initial value, $\beta \sim 0.6$, cannot be recovered without warming up the sample, suggests that the structure of the field-cooled state is altered irreversibly after the onset of motion. We propose that this is due to the introduction of dislocations when, owing to pinningpotential inhomogeneities, some chunks of vortices start moving before others. As was shown in numerical simulations of driven two-dimensional interacting system $\mathrm{s}^{26}$, the dislocations minimize their energy by forming grain boundaries and by aligning their Burgers vectors along the direction of motion. When the drive is suddenly removed they drift to restore the original state. However, if annealing timescales are much longer than experimental times, the grain boundaries coarsen, forming a more entangled network of dislocations, resulting in a lower value of $\beta$.

It is generally accepted that the energy landscape of a finite disordered system has many local minima corresponding to metastable configurations surrounded by high energy barriers that can trap the system ${ }^{8}$. The trapping time in a metastable state increases with trap depth. In this context we can model the 

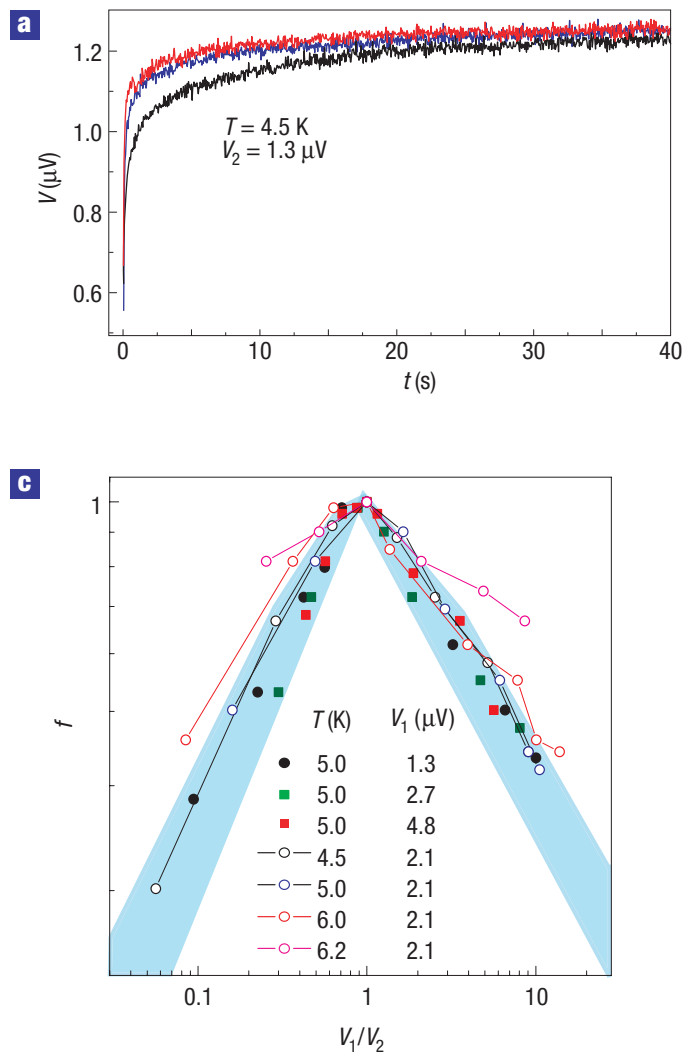
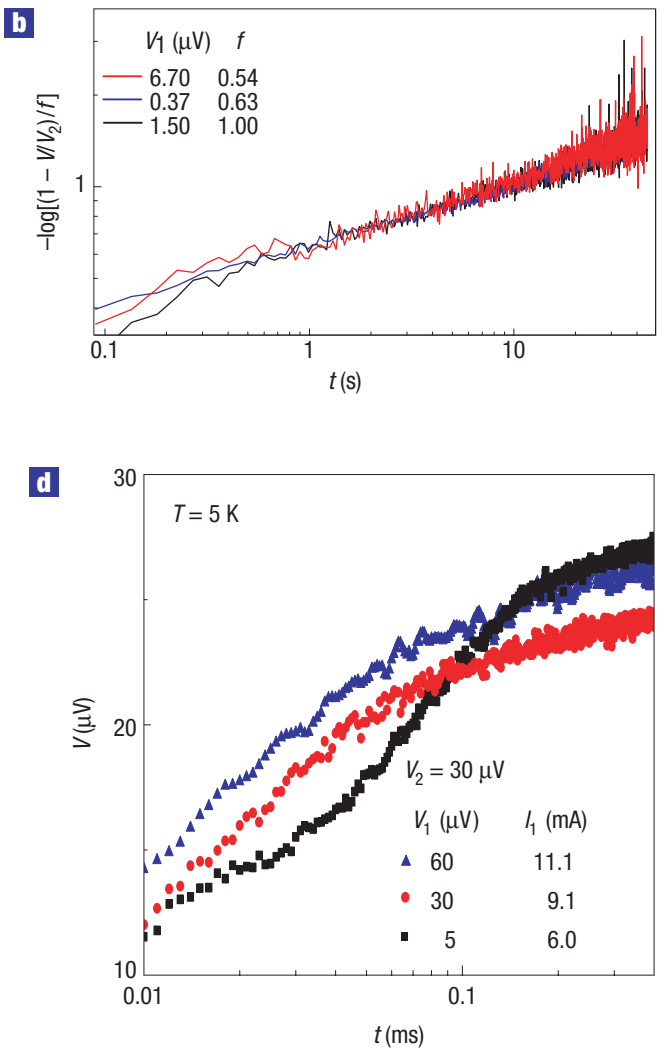

Figure 2 Memory of pulse amplitude. a, Time evolution of the second pulse for $V_{2}=1.3 \mu \mathrm{V}$. $\mathbf{b}$, Same data as in $\mathbf{a}$, showing that there exists a value, $f\left(V_{1} / V_{2}\right)$, for which the scaled data, $-\log \left[\left(1-V / V_{2}\right) / f\right]$, collapse onto a master curve. c, The memory function, $f\left(V_{1} / V_{2}\right)$, obtained as described in $\mathbf{b}$. The highlighted area encloses data taken in the Bragg-glass regime, where memory is strongest. For $T>5.7 \mathrm{~K}$ flattens out, signalling a more feeble memory. $\mathbf{d}$, Response in the first $0.3 \mathrm{~ms}$ of the second pulse, for $t_{\mathrm{w}}=240 \mathrm{~s}, l_{2}=9.1 \mathrm{~mA}$, showing strong first-pulse memory.

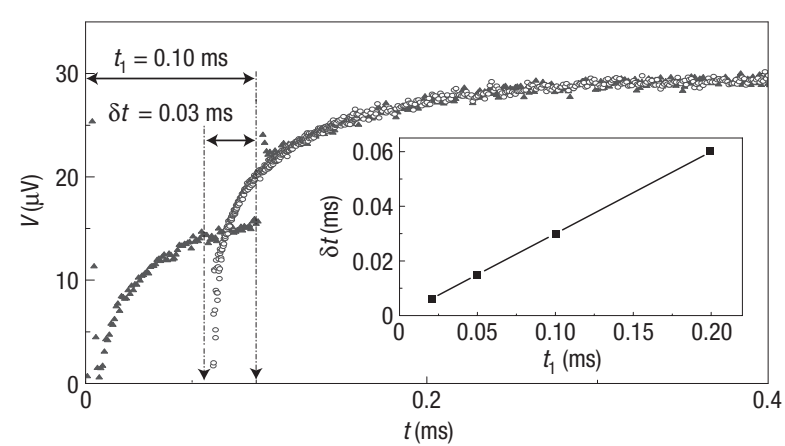

Figure 3 Step-pulse response. Response to step pulse $\left(I_{1}=8.12 \mathrm{~mA}\right.$, $I_{2}=9.13 \mathrm{~mA}, t_{\mathrm{w}}=0$ ). The second-pulse response (triangles) is compared with the response to a single pulse with the same current level $I_{2}$ (circles). The two curves overlap when shifting the time axis by $t_{1}-\delta t$. The inset shows that $\delta t$ is linear in $t_{1}$.

dynamics of the vortex system by mapping each state onto a point in configuration space and representing the evolution between two states by a connecting trajectory consisting of a sequence of trapped states. Thus, during the first pulse the system evolves from the field-cooled state to the moving vortex state along a connecting trajectory as shown in Fig. 4. During $t_{\mathrm{w}}$ when the drive is absent, the system drifts away from the moving-state point towards a lower-energy relaxed state, where the grain boundaries have coarsened. Both simple ageing and the response to a step pulse can be described within this model.

The key point for simple ageing is that the deepest traps encountered during $t_{\mathrm{w}}$ must have trapping times $\tau_{\mathrm{t}} \sim t_{\mathrm{w}}$. This was shown to be the case ${ }^{8}$ for trapping times that have exponential or power-law distributions, provided the maximum trapping time is much shorter than $t_{\mathrm{w}}$. Therefore, during the subsequent second pulse, while the system is driven back towards the moving state and traversing the same deepest trap, the trapping time should again be $\sim t_{\mathrm{w}}$, provided the drive does not significantly change the energy landscape. In other words, $t_{\mathrm{w}}$ selects a timescale (out of a broad distribution) that becomes the characteristic scale for subsequent response events. This naturally gives rise to simple ageing. However, in spite of its 'simplicity', simple ageing is rare and was only recently observed in a Coulomb glass ${ }^{5,6}$ and in a spin glass ${ }^{7}$. It is noteworthy that ageing may disappear altogether if the distribution of $\tau_{\mathrm{t}}$ is not continuous or if it is truncated. For example, in very disordered samples where $\tau_{\mathrm{t}} \gg t_{\mathrm{w}}$, the system remains trapped close to the moving state long after the drive is removed. This is the case for vortex states in Fe-doped $2 \mathrm{H}-\mathrm{NbSe}_{2}$, where no ageing was observed for $t_{\mathrm{w}} \leq 24 \mathrm{~h}$ (ref. 17). At the other extreme lies the case of clean samples, where ageing is not seen either because there is a unique equilibrium state (no trapping) or because $\tau_{\mathrm{t}}$ is much shorter than the measurement times. This implies that there is a critical amount of disorder needed to observe ageing (see the Supplementary Information). 


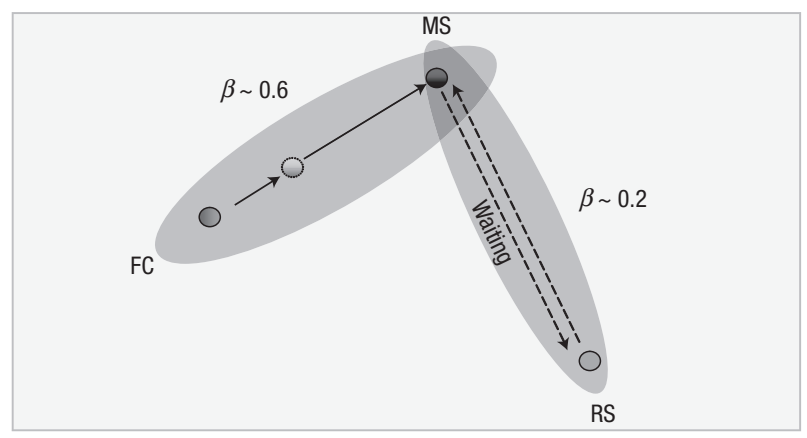

Figure 4 Trajectories in configuration space. Configuration-space representation of vortex states and connecting trajectories. During the first pulse the system evolves along the FC-MS (field-cooled-moving-state) trajectory, which is independent of driving force. In between pulses the system drifts towards the relaxed state (RS). During the second pulse, it is driven back to the moving state.

The response to step pulses imposes two additional constraints. (1) For a given first-pulse amplitude $I$, the configuration space 'speed', $v(I)$, along the FC-MS trajectory is constant. (2) $v(I)$ increases with increasing $I$. Thus during the first pulse the system evolves at an average speed $v_{1}=v\left(I_{1}\right)$ such that at time $t_{1}$ it reaches an intermediate point $\mathrm{P}$ along FC-MS. During the second pulse the remainder of the trajectory is traversed at a higher speed $v_{2}=v\left(I_{2}\right)$. Had the entire FC-MS trajectory been traversed at speed $v_{2}$, then $\mathrm{P}$ would have been reached at a time $\delta t=t_{1}\left(v_{1} / v_{2}\right)$ after the pulse onset. Therefore, the response during the second pulse, $V^{\prime}\left(t-\left(t_{1}-\delta t\right)\right)$, is identical to that for a single pulse of amplitude $I_{2}$ applied $\delta t$ before $t_{1}$.

The experiments described here demonstrate that in the presence of quenched disorder the response of a driven vortex system to a current pulse can be described by KWW time dependence, with the exponent reflecting the deviation of the initial state from equilibrium. It is shown that there exists a range of strengths of the quenched disorder for which the system can show ageing and that simple ageing arises naturally in samples with a continuous distribution of trapping times whose range is much wider than that of experimental waiting times.

Received 31 July 2006; accepted 24 November 2006; published 21 January 2007.

References

1. Cugliandolo, L. F. in Dynamics of Glassy Systems, Lecture Notes in Slow Relaxation and Non Equilibrium Dynamics in Condensed Matter, Les Houches Session 77 July 2002 (eds Barrat, J.-L. Dalibard, J., Kurchan, J. \& Feigel'man, M. V.) Preprint at <http://arxiv.org/abs/cond-mat/0210312>.
2. Struik, L. C. E. Physical Aging in Amorphous Polymers and Other Materials (Elsevier Amsterdam, 1978)

3. Lundgren, L., Svedlindh, P., Nordblad, P. \& Beckman, O. Dynamics of the relaxation-time spectrum in a CuMn spin-glass. Phys. Rev. Lett. 51, 911-914 (1983).

4. Lederman, M., Orbach, R., Hammann, J. M., Ocio, M. \& Vincent, E. Dynamics in spin glasses. Phys. Rev. B 44, 7403-7412 (1991).

5. Vaknin, A. \& Ovadyahu, Z. Aging effects in an Anderson insulator. Phys. Rev. Lett. 84, 3402-3405 (2000).

6. Orlyanchik, V. \& Ovadyahu, Z. Stress aging in the electron glass. Phys. Rev. Lett. 92, 066801 (2004).

7. Rodriguez, G. F., Kenning, G. G. \& Orbach, R. Full aging in spin glasses. Phys. Rev. Lett. 91, 037203 (2003).

8. Bouchaud, J. P. Weak ergodicity breaking and aging in disordered systems. J. Phys. (Paris) I 2 , 1705-1713 (1992)

9. Palmer, R. G., Stein, D. L., Abrahams, E. \& Anderson, P. W. Models of hierarchically constrained dynamics for glassy relaxation. Phys. Rev. Lett. 53, 958-961 (1984).

10. Kohlrausch, R. Theorie des elektrischen rückstandes in der leidener flasche. Ann. Phys. Chem. (Leipzig) 91, 179-214 (1874).

11. Williams, G. \& Watts, D. C. Non-symmetrical dielectric relaxation behavior arising from a simple empirical decay function. Trans. Faraday Soc. 66, 80-85 (1970).

12. Repain, V. et al. Creep motion of a magnetic wall: Avalanche size divergence. Europhys. Lett. 68, 460-466 (2004).

13. Måløy, K. J. \& Schmittbuhl, J. Dynamical event during slow crack propagation. Phys. Rev. Lett. 87, 105502 (2001).

14. Giamarchi, T. \& LeDoussal, P. Elastic theory of flux lattices in the presence of weak disorder. Phys. Rev. B 52, 1242-1245 (1995).

15. Klein, T. et al. Bragg glass phase in the vortex lattice of a type II superconductor. Nature 413, 404-406 (2001).

16. Henderson, W., Andrei, E. Y., Higgins, M. J. \& Bhattacharya, S. Metastability and glassy behavior of a driven flux-line lattice. Phys. Rev. Lett. 77, 2077-2080 (1996).

17. Xiao, Z. L., Andrei, E. Y. \& Higgins, M. J. Flow induced organization and memory of a vortex lattice Phys. Rev. Lett. 83, 1664-1667 (1999).

18. Valenzuela, S. O. \& Bekeris, V. Plasticity and memory effects in the vortex solid phase of twinned $\mathrm{YBa}_{2} \mathrm{Cu}_{3} \mathrm{O}_{7}$ single crystals. Phys. Rev. Lett. 84, 4200-4203 (2000).

19. Li, G., Andrei, E. Y., Xiao, Z. L., Shuk, P. \& Greenblatt, M. Glassy dynamics in a moving vortex lattice. J. Phys. IV 131, 101-107 (2005).

20. Bustingorry, S., Cugliandolo, L. F. \& Domínguez, D. Out-of-equilibrium dynamics of the vortex glass in superconductors. Phys. Rev. Lett. 96, 027001 (2006).

21. Li, G., Andrei, E. Y., Xiao, Z. L., Shuk, P. \& Greenblatt, M. Onset of motion and dynamic reordering of a vortex lattice. Phys. Rev. Lett. 96, 017009 (2006).

22. Shi, A. C. \& Berlinsky, A. J. Pinning and $I-V$ characteristics of a two-dimensional defective flux-line lattice. Phys. Rev. Lett. 67, 1926-1929 (1991).

23. Koshelev, A. E. \& Vinokur, V. M. Dynamic melting of the vortex lattice. Phys. Rev. Lett. 73, 3580-3583 (1994).

24. Balents, L., Marchetti, M. C. \& Radzihovsky, L. Nonequilibrium steady states of driven periodic media. Phys. Rev. B 57, 7705-7739 (1998).

25. LeDoussal, P. \& Giamarchi, T. Moving glass theory of driven lattices with disorder. Phys. Rev. B 57, 11356-11403 (1998).

26. Reichhardt, C. \& Olson Reichhardt, C. J. Coarsening of topological defects in oscillating systems with quenched disorder. Phys. Rev. E 73, 046122 (2006).

\section{Acknowledgements}

We wish to thank E. Abrahams, T. Giamarchi, E. Lebanon, M. Markus, C. Olson Reichhardt, A. Rosch and B. Rosenstein for useful discussions. We thank I. Skachko for technical support. The work was supported by NSF-DMR-0456473 and by DOE DE-FG02-99ER45742.

Correspondence and requests for materials should be addressed to E.Y.A.

Supplementary Information accompanies this paper on www.nature.com/naturephysics.

Author contributions

X.D. and G.L. carried out the experiments and E.Y.A., X.D. and G.L. participated in the analysis. X.D. and G.L. participated in the experimental set-up and the project was conceived by E.Y.A. The crystals were supplied by M.G. and P.S.

Competing financial interests

The authors declare that they have no competing financial interests.

Reprints and permission information is available online at http://npg.nature.com/reprintsandpermissions/ 